\title{
Influence of glenohumeral conformity on glenoid stresses after total shoulder arthroplasty
}

\author{
Alexandre Terrier, PhD, a Philippe Büchler, $\mathrm{PhD},{ }^{a}$ and Alain Farron, MD, ${ }^{b}$ Lausanne, Switzerland
}

Glenohumeral conformity has been reported to be one of the most critical implant-related features that may affect the occurrence of glenoid loosening. This study evaluated the mechanical effects of this parameter with a 3-dimensional finite element model of a prosthetic shoulder, which included the scapula, the humerus, and the rotator cuff muscles. Aequalis humeral and glenoid components were implanted numerically according to manufacturer's recommendations for 2 different orientations of the glenoid component $10^{\circ}$ and $15^{\circ}$ of retroversion). Different values of glenohumeral conformity (1-15 mm of radial mismatch) were tested by a progressive flattening of the glenoid surface. Free and countered rotation movements were simulated. Glenohumeral contact pressure, cement stress, shear stress, and micromotions at the bone-cement interface were calculated. At $\mathrm{O}^{\circ}$ of retroversion, conformity had only a slight effect, whereas at $15^{\circ}$ of retroversion, all quantities increased by more than $200 \%$ and exceeded critical values above $10 \mathrm{~mm}$ of mismatch. (J Shoulder Elbow Surg 2006; 15:515-520.)

Although glenohumeral arthroplasty has proved to be an effective procedure, glenoid loosening is a frequent complication after prosthetic replacement of the shoulder. ${ }^{35,41}$ Among the possible causes, glenohumeral conformity is one of the implant-related features that may affect the occurrence of glenoid loosening. ${ }^{10,31}$

Glenohumeral conformity is usually defined as the difference between the radii of curvature of the glenoid and the humeral head surfaces. It may be related

From the aDivision of Orthopaedic Research, Swiss Federal Institute of Technology, and bHôpital Orthopédique de la Suisse Romande, University of Lausanne

This study was partly supported by Tornier (Montbonnot, France), which also provided the geometry of the prosthetic design used in this work.

Reprint requests: Alexandre Terrier, PhD, Bldg AA-B, Station 15, Orthopedic Research Center, Swiss Federal Institute of Technology, $\mathrm{CH}$ 1015 Lausanne, Switzerland (E-mail:alexandre.terrier@epfl.ch).

Copyright ( 2006 by Journal of Shoulder and Elbow Surgery Board of Trustees.

$1058-2746 / 2006 / \$ 32.00$

doi: 10.1016/i.jse.2005.09.021 to 2 mechanical aspects of the joint: the glenohumeral contact surface and the glenohumeral obligate translations. Basically, conforming designs present a greater contact area and, therefore, a smaller contact pressure, ${ }^{39}$ reducing the stress within implant, cement, and bone, as well as at their interfaces, and finally reducing the risk of wear and fatigue of the polyethylene and cement, as well as the reliability of the bone-cement interface. In parallel, through their natural geometric constraint, conforming designs are assumed to improve joint stability. ${ }^{39}$ On theother hand, conforming implants reduce the natural level of obligate translations between the articular surfaces. $9,12,16-18,28$ These translations are constrained by eccentric forces, which may create excessive rim stress $^{15,31}$ and may lead to implant loosening. ${ }^{5}$ Therefore, because glenohumeral conformity is related to opposite mechanisms, an ideal value may be hypothesized. Finally, it seems obvious that the stress pattern at the glenohumeral interface influences the longevity of the joint replacement with respect to stability, loosening of the glenoid component, and wear of the components.

From cadaveric studies, it was found that a radial mismatch of $4 \mathrm{~mm}$ best reproduced the glenohumeral translation. ${ }^{13,16}$ Collins et a $\left.\right|^{5}$ recommended 3 to 5 $\mathrm{mm}$ of mismatch, whereas Walch et al, ${ }^{39}$ in a retrospective multicenter clinical study, recommended 6 to $10 \mathrm{~mm}$. Friedman ${ }^{7}$ emphasized the risk of polyethylene fracture with radial mismatch exceeding $10 \mathrm{~mm}$. However, despite several clinical, 11,39 experimental, ${ }^{10,13,31}$ and numerical studies, ${ }^{21,33}$ the mechanical effects of glenohumeral conformity are not yet completely understood, 6,16 and recommendations for an ideal mismatch are still uncertain. ${ }^{39}$

Therefore, the aim of this study was to evaluate the influence of glenohumeral conformity on glenoid stress by use of a finite element model of a prosthetic shoulder. In addition, because retroversion of the glenoid component is often reported, ${ }^{38}$ this parameter was included in this study. For that reason, a rotation movement was chosen for this analysis, because it was more appropriate to reveal critical joint contacts caused by the coupled effect of conformity and retroversion. 


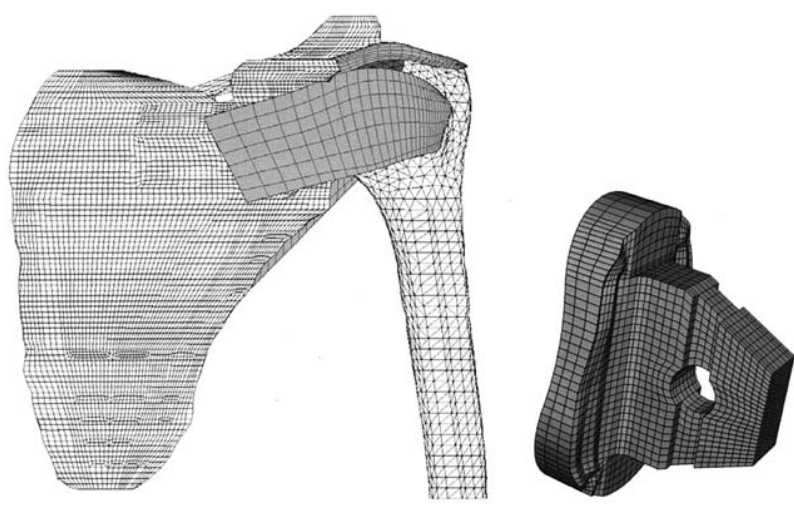

Figure 1 Finite element mesh of prosthetic shoulder and detail view of glenoid component.

\section{MATERIALS AND METHODS}

A fresh-frozen cadaveric shoulder without any evidence of pathology was scanned every $1 \mathrm{~mm}$, from the acromion to the humeral midshaft. Thereafter, careful dissection was performed to measure (Fastrak stylus, Polhemus Inc., www. polhemus.com) the exact insertion zones of the major rotator cuff muscles: infraspinatus, supraspinatus, and subscapularis. ${ }^{2,3}$ The 3-dimensional geometry of the scapula and humerus was obtained from computed tomography segmentation, whereas the muscles were reconstructed from the measured insertions ${ }^{2,3}$ and general anatomic considerations. Humeral and glenoid components (Aequalis, Tornier, Montbonnot, France) were implanted numerically into the virtual shoulder in 2 different glenoid orientations: $0^{\circ}$ and $15^{\circ}$ of retroversion. The glenoid component was an all-polyethylene, keeled, and flat-back design (Figure 1). It was surrounded by a uniform cement layer of $0.5 \mathrm{~mm}$ (recommended by the manufacturer). The material property of the glenoid, cement, and scapula was linear elastic. ${ }^{3}$ The elastic modulus of the scapula was related to bone density ${ }^{34}$ which was derived from computed tomography. The muscles were characterized by hyperelastic law, based on an exponential strain energy potential. ${ }^{26}$ The humerus and humeral component were rigid. The implant-cement interface was perfectly bonded, whereas the Coulomb friction law governed the bone-cement interface. The friction coefficient $(\mu \mathrm{m}, 0.6)$ corresponded to a cement-compact bone interface. ${ }^{29,40}$ The scapula was fixed at the insertion points of the trapezius and rhomboid muscles and at some points of its anterior side, corresponding to contact with the thorax. The movements of the humerus were restricted by fixing the elbow and avoiding abduction/adduction and flexion/ extension. Rotation was simulated by the direct action of the muscles, starting from a position of neutral rotation and abduction of the arm, when the humeral component faced the glenoid component. In a first step, the humerus was fixed, and all muscles were pretensioned with a $I=N$ force. Then, the humerus was released, and the glenohumeral contact was achieved. Finally, a displacement of the scapular extremity of the subscapularis was imposed to generate $60^{\circ}$ of internal rotation; in the same way, a displacement of the infraspinatus extremity generated external rotation. In addition, a countered movement was obtained by attaching

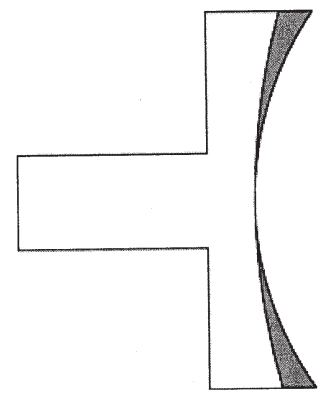

Figure 2 The variation in glenohumeral conformity was obtained by a progressive flattening of the spherical glenoid surface (gray zone in this horizontal view), maintaining its central point on a fixed position.

an axial spring to the humerus, in the diaphyseal direction. This spring passively induced an increasing torque on the humerus as rotation occurred and corresponded to about $10 \mathrm{~N}$ of traction in the hand (the forearm being perpendicular to the arm) at $60^{\circ}$ of rotation.

Glenohumeral conformity variations were obtained by a progressive flattening of the glenoid surface to reproduce 7 values of radial mismatch: $1,3.5,6,8.5,10,12$, and 15 $\mathrm{mm}$ (Figure 2). For each of these glenoid components, free and countered rotations were simulated, with and without retroversion. Different mechanical variables were calculated: the glenohumeral contact pressure, the cement stress, the shear stress, and relative micromotions at the bonecement interface. Finite element analyses and postprocessing were achieved with the Abaqus software suite (Abaqus Inc, http://www.abaqus.com.

\section{RESULTS}

During the full range of motion, the results followed the same trend in internal rotation as in external rotation. However, the effect was maximal at $60^{\circ}$ of internal rotation. Therefore, the calculated quantities were only presented at this extreme position. Spatial distributions (Figures 3 and 4 ) and maximal values (Figure 5) were considered.

\section{Glenohumeral contact pressure}

Glenohumeral contact pressure increased by about $300 \%$ as mismatch increased from 1 to $15 \mathrm{~mm}$, independent of the retroversion angle or the countered force. The contact pressure was amplified by $10 \%$ to $20 \%$ when retroversion was added and increased drastically from $12 \mathrm{~mm}$ of mismatch when the countered force and retroversion were combined (black curve, top right graph of Figure 5). The contact position remained symmetrically centered on the glenoid surface in the free/no-retroversion case, but it moved posteriorly with the countered force or with retroversion (Figure 3). When the countered force and retroversion were combined, the contact point reached the posterior rim of the glenoid surface. 

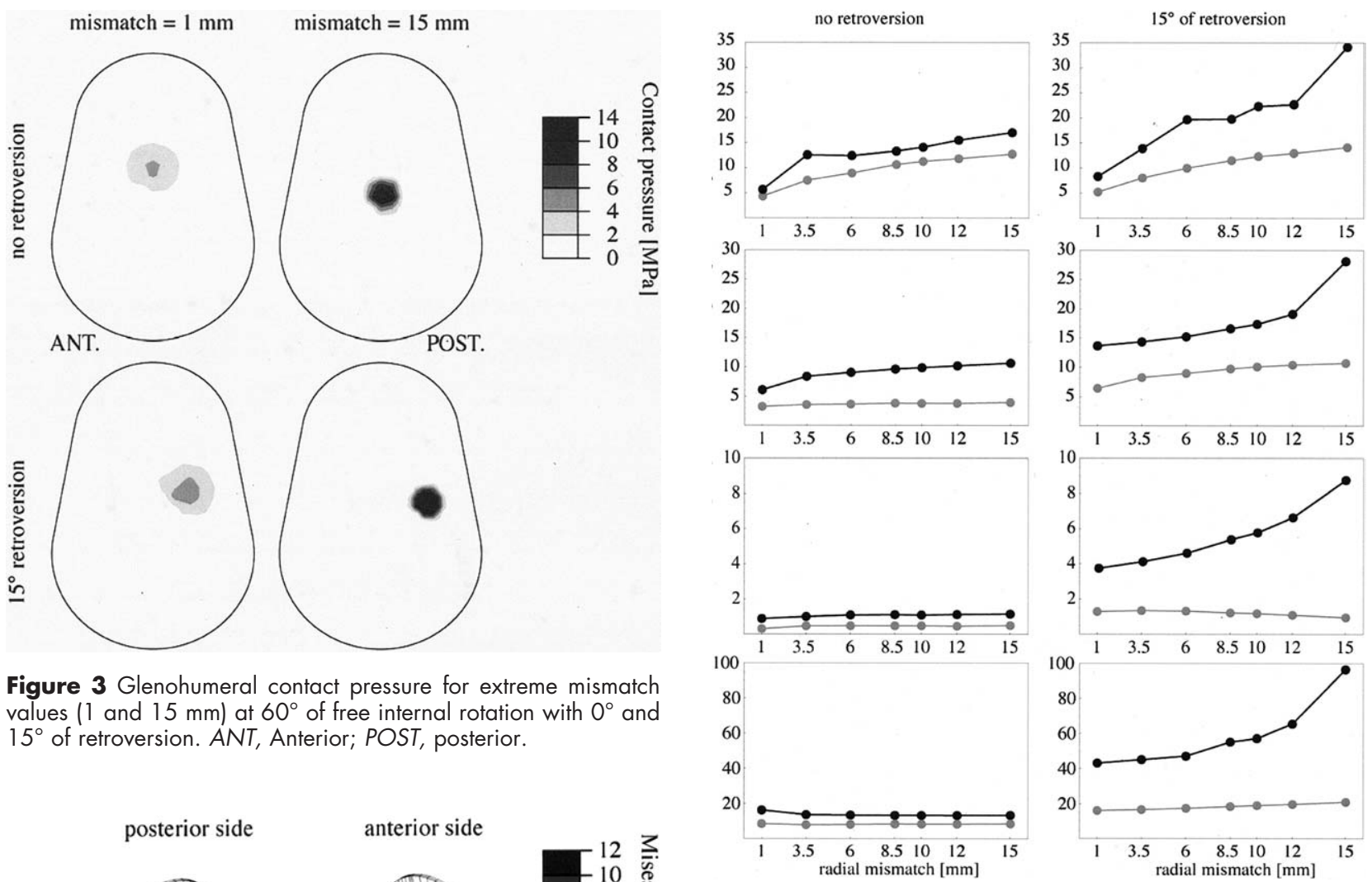

Figure 3 Glenohumeral contact pressure for extreme mismatch values $\left(1\right.$ and $15 \mathrm{~mm}$ ) at $60^{\circ}$ of free internal rotation with $0^{\circ}$ and $15^{\circ}$ of retroversion. ANT, Anterior; POST, posterior.
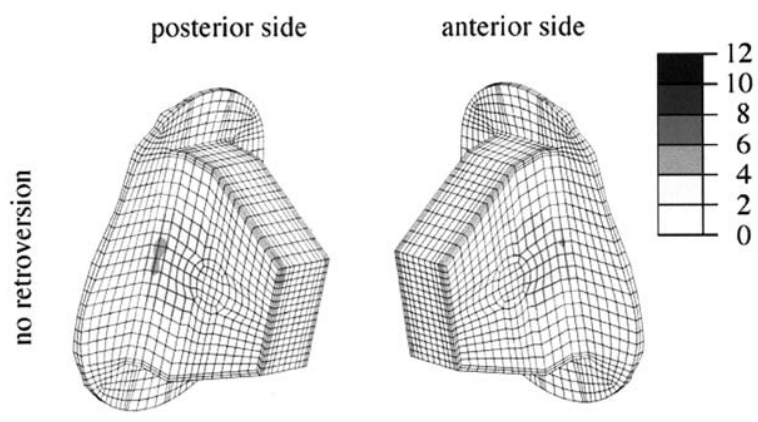

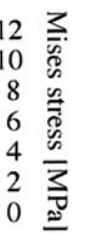
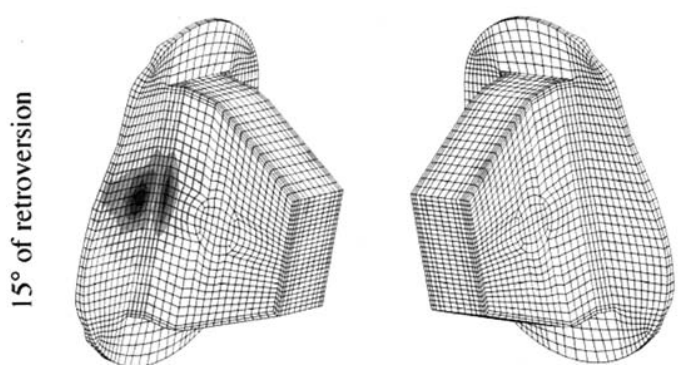

Figure 5 Maximal value of glenohumeral contact pressure, cement von Mises stress, bone- cement interfacial shear stress, and bone-cement interfacial micromotions at $60^{\circ}$ of free (gray) and countered (black) internal rotation with $0^{\circ}$ (left) and $15^{\circ}$ (right) of retroversion.

came important in the countered/retroversion case and also severe above $12 \mathrm{~mm}$ of mismatch in the countered/retroversion case. As for contact pressure, the stress distribution was symmetric and centered in the free/no-retroversion case but moved posteriorly in the countered or retroversion case (Figure 4).

\section{Bone-cement interfacial shear stress}

Bone-cement interfacial shear stress was almost not sensitive to mismatch except in the countered/retroversion case, where it increased exponentially. It was about 10 times higher in the countered/retroversion case compared with all other cases. At $0^{\circ}$ of retrover-

Figure 4 von Mises stress in cement layer for $15 \mathrm{~mm}$ of mismatch at $60^{\circ}$ of free internal rotation with $0^{\circ}$ and $15^{\circ}$ of $15^{\circ}$ of retroversion.

\section{Cement stresses}

A similar behavior was observed in the cement layer. Indeed, von Mises stress increased in all cases by about $200 \%$ as mismatch increased. This increase was slight in the free/no-retroversion case but besion, its peak value was located near the keel-plate edges; it moved behind the keel tip when the glenoid was retroverted by $15^{\circ}$.

\section{Bone-cement relative micromotions}

Without retroversion, the bone-cement relative micromotions were almost not influenced by mismatch. Conversely, at $15^{\circ}$ of retroversion, micromotions increased as mismatch increased. This increase was 
only important with the countered force and, in this case, increased rapidly above $10 \mathrm{~mm}$ of mismatch.

\section{DISCUSSION}

Glenohumeral conformity is reported as one of the implant features that might influence glenoid loosening after total shoulder arthroplasty. Because findings from the clinical and biomechanical studies on this topic are still unclear, there is a need to investigate this feature further. In this study, the effect of this parameter was analyzed in parallel with glenoid retroversion. Indeed, the angle of retroversion of the glenoid component is often difficult to correct or to set precisely during the surgical procedure, and it also modifies the glenoid articular surface concurrently with glenohumeral conformity. Because glenoid retroversion mainly occurs in the horizontal plane, a rotation movement was more appropriate to reveal critical joint contact stresses. Therefore, a rotation movement was simulated by means of a 3dimensional finite element model of a shoulder to analyze the mechanical effect of glenohumeral conformity on sensitive aspects of glenoid loosening (ie, glenohumeral contact pressure, von Mises stress within the cement, shear stress, and micromotions at the bone-cement interface). These variables were chosen because they might be related to polyethylene wear, cement cracks, and reliability of the bonecement interface.

Our results showed that, as mismatch increased, glenohumeral contact pressure increased significantly (300\% from 1 to $15 \mathrm{~mm})$, and as a result, stress within the polyethylene increased, as reported by other studies. ${ }^{33}$ The contact pressure was maximal in the countered or retroversion case. From $10 \mathrm{~mm}$ of mismatch, it exceeded the polyethylene yield strength, 20,36 and consequently, damage accumulation and a reduced fatigue life of the component could be expected. Moreover, because contact pressure is related to wear, we can assume that a mismatch increase will also produce a wear increase.

Cement stress also increased as mismatch increased; however, the cement stress increase was only severe for the countered or retroversion case and above $10 \mathrm{~mm}$ of mismatch. This value of $10 \mathrm{~mm}$ was already reported as a limit value for radial mismatch. ${ }^{7}$ Except for the free/no-retroversion case, cement stresses were high enough (>5-7 MPa) to induce cement failure. ${ }^{15}$ This result indicates that the stress within the cement is an important point to account for in designing shoulder implants or implantation techniques.

In a cadaveric study, Nyffeler et a ${ }^{24}$ measured the pullout strength of cemented glenoid component pegs for different surfaces and macrostructures. Because failure occurred at the polyethylene-cement interface for all cases except one loccurring at the bone-cement interface), the maximum pullout strength can be used to minimize the failure strength of the bone-cement interface. This rough estimate gives a failure strength of at least $3 \mathrm{MPa}$, which is in the range of reported values $(2-12 \mathrm{MPa})$ of shear strength at the bonecement interface. ${ }^{22}$ In the countered or retroversion case, this value was exceeded at the tip of the keel. This may suggest that debonding begins at this location.

Above $10 \mathrm{~mm}$ of mismatch, in the countered/ retroversion case, micromotions reached a reported limit for the formation of fibrous tissue, ${ }^{14}$ which is known to promote implant loosening. Moreover, when the implant starts to debond, cement particles, resulting from microfractures, may migrate toward regions of high slipping and accentuate the effect of the micromotions on fibrous tissue formation. ${ }^{1}$

In a cadaveric study with various glenoid designs, Severt et a ${ }^{\beta 1}$ reported that reaction forces to fixed displacements of the humeral head were higher in conforming designs. In the same way, Walch et al, ${ }^{39}$ in a retrospective multicenter clinical study of the Aequalis implant, reported that a radial mismatch of less than $6 \mathrm{~mm}$ induced higher radiolucency scores and concluded that, for this implant, the ideal radial mismatch should range between 6 and $10 \mathrm{~mm}$. Conversely, in a clinical study on retrieved glenoid components. Hertel and Ballmer ${ }^{11}$ observed that the newly formed concavity of the glenoid component due to wear perfectly matched the radius of the prosthetic head. These observations confirmed those of other researchers ${ }^{8,30}$ and assume that full articular conformity would be preferable.

The present model has some limitations. First, it is based on a single shoulder, having no degenerative alterations, instead of multiple arthritic shoulders, which usually present degenerative changes such as posterior glenoid erosion. This method may, however, be valid to analyze the general biomechanical effects and trends of the phenomenon. Moreover, it should be noted that, providing a correct description of the different material properties, the finite element technique is commonly accepted for calculating the motion and stress state of deformable structures without further experimental assessments. ${ }^{23,25,32}$ The cement layer surrounding the glenoid was uniform, and the contact law did not include a stress failure criterion in tension, accounting for adhesion of the cement to the bone. Only rotations were simulated in this work, inducing lower contact forces and stresses than abduction. Nevertheless, by use of an axial spring, simulating a countered movement, the glenohumeral contact force reached $250 \mathrm{~N}$, which is between one half and one third of the maximal abduction force. ${ }^{19,27,37}$ However, although large translations of the humeral head were observed, the expected in- 
crease in interface stresses associated with a conformity increase ${ }^{15,39}$ was not observed. In our opinion, this phenomenon is mainly caused by tangential forces associated with important forces of the stabilizer muscles, which occur, for instance, during standing up, pushing, pulling, or carrying loads. Despite these limitations, our model can be used to obtain minimum conditions, at least valid in the case of countered rotation, which is a relatively frequent movement during activities of daily living.

In summary, all mechanical variables that were related to bone-cement interface reliability were altered by a decrease in conformity. Without retroversion, the glenohumeral contact forces were almost centered and aligned to the implant axis. With retroversion, the contact forces became eccentric, increasing the stresses at the posterior part of the implantcement-bone complex. Finally, although an ideal mismatch could not be established, we confirm that radial mismatch above $10 \mathrm{~mm}$ should be avoided.

The clinical conclusion of this study confirms the importance of glenoid component orientation and, thus, the importance of the surgeon's ability to reproduce the natural glenohumeral joint. Moreover, we conclude that posterior wear of the glenoid, uncorrected with a prosthesis implanted in retroversion, may accelerate the mechanisms of glenoid loosening. 4

\section{REFERENCES}

1. Aspenberg P, Herbertsson P. Periprosthetic bone resorption. Particles versus movement. J Bone Joint Surg Br 1996;78:64 1-6.

2. Buchler P, Rakotomanana L, Farron A. Virtual power based algorithm for decoupling large motions from infinitesimal strains: application to shoulder joint biomechanics. Comput Methods Biomech Biomed Engin 2002;5:387-96

3. Buchler $P$, Ramaniraka NA, Rakotomanana LR, lannotti JP, Farron A. A finite element model of the shoulder: application to the comparison of normal and osteoarthritic joints. Clin Biomech 2002; 17:630-9.

4. Büchler $P$, Terrier A, Farron A. Influence of the glenoid version on shoulder implant loosening - a finite element analysis. J Shoulder Elbow Surgery. In press 2006

5. Collins D, Tencer A, Sidles J, Matsen F III. Edge displacement and deformation of glenoid components in response to eccentric loading. The effect of preparation of the glenoid bone. J Bone Joint Surg Am 1992;74:501-7.

6. Flatow El. Prosthetic design considerations in total shoulder arthroplasty. Semin Arthroplasty 1995;6:233-44.

7. Friedman JR. Glenohumeral translation after total shoulder arthroplasty. J Shoulder Elbow Surg 1992; 1:312-6.

8. Gunther SB, Graham J, Norris TR, Ries MD, Pruitt L. Retrieved glenoid components: a classification system for surface damage analysis. J Arthroplasty 2002; 17:95-100.

9. Harryman DT II, Sidles JA, Harris SL, Matsen FA III. The role of the rotator interval capsule in passive motion and stability of the shoulder. J Bone Joint Surg Am 1992;74:53-66.

10. Harryman DT, Sidles JA, Harris SL, Lippitt SB, Matsen FA III. The effect of articular conformity and the size of the humeral head component on laxity and motion after glenohumeral arthroplasty. A study in cadavera. J Bone Joint Surg Am 1995;77:555-63.
1 1. Hertel R, Ballmer FT. Observations on retrieved glenoid components. J Arthroplasty 2003; 18:36 1-6.

12. Howell SM, Galinat BJ, Renzi AJ, Marone PJ. Normal and abnormal mechanics of the glenohumeral joint in the horizontal plane. J Bone Joint Surg Am 1988;70:227-32.

13. lannotti JP, Gabriel JP, Schneck SL, Evans BG, Misra S. The normal glenohumeral relationships. An anatomical study of one hundred and forty shoulders. J Bone Joint Surg Am 1992;74:49 1500.

14. Jasty M, Bragdon C, Burke D, O'Connor D, Lowenstein J, Harris $\mathrm{WH}$. In vivo skeletal responses to porous-surfaced implants subjected to small induced motions. J Bone Joint Surg Am 1997;79: 707-14.

15. Karduna AR, Williams GR, lannotti JP, Williams JL. Total shoulder arthroplasty biomechanics: a study of the forces and strains at the glenoid component. J Biomech Eng 1998; 120:92-9.

16. Karduna AR, Williams GR, Williams JL, lannotti JP. Glenohumeral joint translations before and after total shoulder arthroplasty. A study in cadavera. J Bone Joint Surg Am 1997;79: $1166-74$

17. Karduna AR, Williams GR, Williams JL, lannotti JP. Joint stability after total shoulder arthroplasty in a cadaver model. J Shoulder Elbow Surg 1997;6:506-1 1 .

18. Karduna AR, Williams GR, Williams JL, lannotti JP. Kinematics of the glenohumeral joint: influences of muscle forces, ligamentous constraints, and articular geometry. J Orthop Res 1996; 14:98693.

19. Karlsson D, Peterson B. Towards a model for force predictions in the human shoulder. J Biomech 1992;25: 189-99.

20. Kurtz SM, Pruitt L, Jewett CW, Crawford RP, Crane DJ, Edidin AA The yielding, plastic flow, and fracture behavior of ultra-high molecular weight polyethylene used in total joint replacements. Biomaterials 1998; 19:1989-2003.

21. Lacroix D, Prendergast PJ. Stress analysis of glenoid component designs for shoulder arthroplasty. Proc Inst Mech Eng [H] 1997; $211: 467-74$

22. MacDonald W, Swarts E, Beaver R. Penetration and shear strength of cement-bone interfaces in vivo. Clin Orthop Relat Res 1993:283-8

23. Murphy LA, Prendergast PJ, Resch H. Structural analysis of an offset-keel design glenoid component compared with a centerkeel design. J Shoulder Elbow Surg 2001; 10:568-79.

24. Nyffeler RW, Anglin C, Sheikh R, Gerber C. Influence of peg design and cement mantle thickness on pull-out strength of glenoid component pegs. J Bone Joint Surg Br 2003;85:748-52.

25. Orr TE, Carter DR, Schurman DJ. Stress analyses of glenoid component designs. Clin Orthop Relat Res 1988:217-24.

26. Pioletti DP, Rakotomanana LR. Non-linear viscoelastic laws for soft biological tissues. Eur J Mech A/Solids 2000; 19:749-59.

27. Poppen NK, Walker PS. Forces at the glenohumeral joint in abduction. Clin Orthop Relat Res 1978: 165-70.

28. Poppen NK, Walker PS. Normal and abnormal motion of the shoulder. J Bone Joint Surg Am 1976;58: 195-201.

29. Ramaniraka NA, Rakotomanana LR, Leyvraz PF. The fixation of the cemented femoral component. Effects of stem stiffness, cement thickness and roughness of the cement-bone surface. J Bone Joint Surg Br 2000;82:297-303

30. Scarlat MM, Matsen FA III. Observations on retrieved polyethyene glenoid components. J Arthroplasty 2001;16:795-801.

31. Severt R, Thomas BJ, Tsenter MJ, Amstutz HC, Kabo JM. The influence of conformity and constraint on translational forces and frictional torque in total shoulder arthroplasty. Clin Orthop Relat Res 1993; 151-8

32. Stone KD, Grabowski لע, Cofield RH, Morrey BF, An KN. Stress analyses of glenoid components in total shoulder arthroplasty. J Shoulder Elbow Surg 1999;8:151-8. 
33. Swieszkowski W, Bednarz P, Prendergast PJ. Contact stresses in the glenoid component in total shoulder arthroplasty. Proc Inst Mech Eng [H] 2003;217:49-57.

34. Terrier A, Rakotomanana RL, Ramaniraka AN, Leyvraz PF. Adaptation models of anisotropic bone. Comput Methods Biomech Biomed Engin 1997; 1:47-59.

35. Torchia ME, Cofield RH, Settergren CR. Total shoulder arthroplasty with the Neer prosthesis: long-term results. J Shoulder Elbow Surg 1997;6:495-505.

36. Urries I, Medel FJ, Rios R, Gomez-Barrena E, Puertolas JA. Comparative cyclic stress-strain and fatigue resistance behavior of electron-beam- and gamma-irradiated ultrahigh molecular weight polyethylene. J Biomed Mater Res B Appl Biomater 2004; 70: $152-60$
37. van der Helm FC, Pronk GM. Three-dimensional recording and description of motions of the shoulder mechanism. J Biomech Eng $1995 ; 1$ 17:27-40.

38. Walch G, Badet R, Boulahia A, Khoury A. Morphologic study of the glenoid in primary glenohumeral osteoarthritis. J Arthroplasty $1999 ; 14: 756-60$

39. Walch G, Edwards TB, Boulahia A, Boileau P, Mole D, Adeleine $P$. The influence of glenohumeral prosthetic mismatch on glenoid radiolucent lines: results of a multicenter study. J Bone Joint Surg Am 2002;84:2186-91

40. Walker PS. Human joints and their artificial replacements. Springfield: Charles C.Thomas; 1977.

41. Wirth MA, Rockwood CA Jr. Complications of total shoulder-replacement arthroplasty. J Bone Joint Surg Am 1996;78:603-16. 